\title{
Modelling context: an activity theory approach
}

\author{
Manasawee Kaenampornpan and Eamonn O’Neill \\ Department of Computer Science \\ University of Bath \\ Bath BA2 7AY \\ UK \\ \{cspmk, eamonn\}@Cs.bath.ac.uk
}

\begin{abstract}
In this paper, we review different context classification systems that have been used to define elements of context. Although existing classification systems cover various types of context, in the development of context aware applications, only a few types of context have been used. In this work, we aim to build a context classification model based on Activity Theory that provides a basis both for dialogue amongst context awareness researchers and for the implementation of a context awareness architecture.
\end{abstract}

\section{Introduction}

In an ambient computing environment, the users are able to do their everyday life activities and at the same time access information or use computing services across multiple places and times. As a result, the user's attention may be divided between several simultaneous activities. Moreover, ambient devices are becoming smaller to disappearing, resulting in usability issues. Researchers have attempted to improve user interaction through the notion of context awareness by exploiting information relating to users, devices and environments.

Researchers in the context awareness field produce different definitions and classification systems for context, covering various elements of context. For the most part, however, context aware applications have utilized only isolated subsets of their context, such as a location or a device's state. A truly context aware system needs to take account of the wide range of interrelated types of context and the relationships amongst them. As a precursor to implementing such systems, we need an approach to modelling context that takes account of this complexity.

This paper starts by providing a review of some context classification systems and examples of projects. Secondly some problems with previous context classification systems are discussed. Activity Theory is then introduced as a potentially valuable approach to developing a comprehensive context classification with an example scenario used to demonstrate how different types of context and their relationships may be identified. Finally, we discuss the potential of applying Activity Theory in developing a comprehensive context classification. 


\section{Related Work}

Researchers have tried to develop better understandings of context by producing context definitions and classification systems. Table 1 shows that different researchers have presented context classification systems containing different elements. The columns in Table 1 are derived from elements that researchers have tried to classify as part of context and the rows show different classification systems.

Table 1. Past context classification systems

\begin{tabular}{|c|c|c|c|c|c|c|c|c|}
\hline & Location & \begin{tabular}{|l|} 
Conditions \\
\end{tabular} & $\begin{array}{l}\text { Infrastructure } \\
\text { (Computing } \\
\text { Environment) }\end{array}$ & \begin{tabular}{|c|} 
Information on \\
User
\end{tabular} & Social & $\begin{array}{c}\text { User } \\
\text { Activity }\end{array}$ & Time & $\begin{array}{c}\text { Device } \\
\text { Characteristics }\end{array}$ \\
\hline [Benerecetti et al.'01] & \multicolumn{2}{|c|}{ Physical Environment } & & \multicolumn{2}{|c|}{ Cultural Context } & & & \\
\hline [Schmidt et al.'99] & \multicolumn{3}{|c|}{ Physical Environment } & \multicolumn{3}{|c|}{ Human Factor } & $\bar{x}$ & \\
\hline [Lieberman and Selker'00] & \begin{tabular}{|c|} 
User \\
Environment
\end{tabular} & \begin{tabular}{|c|} 
Physical \\
Environment
\end{tabular} & $\mathrm{x}$ & $\begin{array}{c}\text { User } \\
\text { Environment }\end{array}$ & & & $\mathrm{x}$ & \\
\hline [Hull et al.'97] & & \begin{tabular}{|c|} 
Physical \\
Environment
\end{tabular} & & $x$ & & & & $\mathrm{x}$ \\
\hline [Chalmers and Sloman'99] & $x$ & & $x$ & & $x$ & $x$ & & $x$ \\
\hline [Lucas'01] & \multicolumn{2}{|c|}{ Physical Environment } & \multicolumn{5}{|c|}{ Information context } & $x$ \\
\hline [Schilit et al'94] & \multicolumn{2}{|c|}{ Physical Environment } & $x$ & \multicolumn{2}{|c|}{ User environment } & & & \\
\hline [Abowd and Dey'99] & $x$ & & & Identity & & $x$ & $x$ & Identity \\
\hline [Chen \& Kotz'00] & \multicolumn{8}{|c|}{ Active/Passive } \\
\hline
\end{tabular}

In the first row of Table 1, Benerecetti, Bouquest and Bonifacio [2] have classified context into physical context and cultural context. Physical context is a set of features of the environment while cultural context includes user information, the social environment and beliefs. Schmidt et al [14] have extended the classification into three dimensions: physical environment, human factors and time. The human factors cover the same features as cultural context. They added time because time allows the context model to hold the history of context, which has influence on modelling the user's past, current, and future action.

Lieberman and Selker [12] have ignored time and classified context to include the physical environment, the user environment and the computing environment. In this case, the user environment includes the user's location and is treated separately from the physical environment. Lieberman and Selker treat the computing environment as a separate entity here because they believe that information such as network availability can be of interest to the user and related computing devices. Hull et al [9], Lucas [13] and Chalmers and Sloman [4] argue that characteristics of the device itself, such as screen size and input device, are also of interest to the user and system. They have therefore defined device characteristics as one element of their context classification. Chalmers and Sloman have also added user activity into their context classification. However, they have ignored time and other user characteristics, which may be important elements of context.

Dey [6] has provided a top-level classification system, which includes four types of context: location, identity, time and activity. Dey claims that these are primary types of context that can be used to refer to other secondary context. However, with this classification, there is no clear separation between device and user. The computing 
device and user should be treated differently because they have different features and they affect user behaviour differently. Moreover, the primary context could lead to complications in computing process because the system has to spend time in translating the primary context to secondary context before they can use it in the context aware system.

The classification systems mentioned above are intended to be context models defining what elements of context should be used to reason about the user in order to have a better understanding about the user's intentions. Chen and Kotz [5] have introduced a classification system with a completely different aim where context is classified depending on how it is being used in the application. They have classified context very broadly into two types: active and passive where active context is that which influences the behaviours of an application, and passive context is that which is relevant but not critical to an application.

\section{Analysis of the Problem}

Table 1 shows that there is a multitude of context classification systems, all of which are partial, covering both similar and different elements. Therefore the first problem in the context awareness field is that it lacks a single model of context for designers to refer to so that they have the same understanding of context and understand what key elements should be taken into account in order to have a better understanding of users' behaviour.

Another problem is that in the implementation process, context aware applications have utilized only isolated subsets of their context, such as a location or a device's state, e.g. [1]. There has been little research exploring the relationships between different elements of context and how these relationships can affect the efficiency of context aware applications. These relationships are important in order to use context to represent the world of the user and to help the system better to understand the user's activities and intentions, acknowledging that humans assimilate multiple items of information to perform everyday tasks.

\section{Activity Theory}

\subsection{Why Activity Theory?}

Our main goal of building a context classification system is so that it can be used to build a conceptual model of a user's activity, state and intentions. There are many approaches to analyzing and understanding human activity or tasks, such as Activity Theory [8] and Task Analysis [7, 10]. For the purpose of classifying context and attempting to relate existing partial classifications of context, we have developed an 
approach based on Activity Theory because it has the main characteristics described below.

It Provides a Standard Form for Describing Human Activity. Humans cannot fully understand the full moment-to-moment richness of other humans' activities, states, goals and intentions. Yet they manage successfully and fluently to interact in many highly contextualised ways. Hence, in attempting to produce better contextaware systems, it is neither possible nor necessary to model all the richness of human activity. To make progress from the current state of the art, we propose that a sufficiently comprehensive context classification may be developed using the relatively simple standard form offered by Activity Theory that covers the key elements that have an influence on human activity.

It Provides a Standard Form for Describing Human Activity. Humans cannot fully understand the full moment-to-moment richness of other humans' activities, states, goals and intentions. Yet they manage successfully and fluently to interact in many highly contextualised ways. Hence, in attempting to produce better contextaware systems, it is neither possible nor necessary to model all the richness of human activity. To make progress from the current state of the art, we propose that a sufficiently comprehensive context classification may be developed using the relatively simple standard form offered by Activity Theory that covers the key elements that have an influence on human activity.

It Relates Individual Human Activity to Society. In an ambient computing world, users are not isolated workers at a desktop, in an office. Users are using the computing services within society and that society will have an influence on the user's activities. Therefore, the context classification should allow the system to take account of what can have an impact on human activity within society. Activity Theory explicitly takes society into account in its modelling.

It Provides a Concept of Tool Mediation. Ambient computing users may use several devices or computing services at any time or place. Therefore their tools and the environment are changing all the time. Characteristics of tools have an influence on users' activity. Activity Theory includes this in the model.

It Maps the Relationships Amongst the Elements of a Human Activity Model. Activity Theory also maps the relationships amongst each element that it identifies as having an influence on human activity. This provides us with a potentially useful way to classify context and may be used to model the relationships between each element of context.

\subsection{Background}

Activity Theory was developed by Russian psychologists Vygotsky, Rubinshtein, Leont'ev and others beginning in the 1920s [11]. Activity Theory is a philosophical framework used to conceptualize human activities. In 1987 Engeström [8] proposed a triangular structure of human activity as shown in Figure 1.

The main concepts of this model are: 
Subject: Information about the individual or subgroup chosen as the point of view in the analysis.

Tools: Information about tools, which can mean either psychological or physical tools.

Community: Information about individuals or subgroups who share the same object.

Division of labour: The division of tasks between members of the community.

Rules: Explicit or implicit regulations, norms and conventions that constrain action or interaction.

Object: Target of the activity within the system: subject's intention or objective.

Outcome: The result when the object is met.

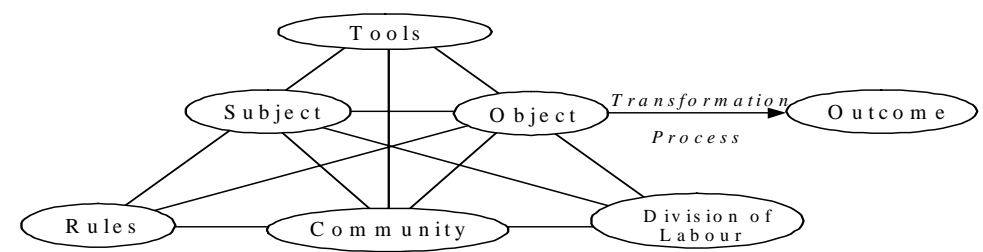

Figure 1. The full structure of Activity Theory introduced by Engeström

Activity Theory describes and relates key elements that influence human activity. However, applying Activity Theory to provide a context classification model that covers all possible contexts in an ambient computing world is not a simple process. Further work is needed to develop a context classification model that can be used as a framework to interpret the context of user behaviour in a context aware system.

\subsection{Example Scenario}

In moving from Activity Theory to a context classification model, we have adopted a scenario-based approach [3]. Scenarios are used to help identify key elements and how they influence user activity. By way of illustration here, we provide a brief account of an example scenario.

Henry is a new PhD student. He is assigned to teach once a week on Tuesday 9.15$10.15 \mathrm{am}$. On Tuesday at 9.15, he arrives at the teaching room and discovers that he has forgotten the lecture notes. Thus, he has to try to remember what his notes contained and reproduce them. In the end, he gives up and spends 15 minutes fetching the lecture notes from his office. 


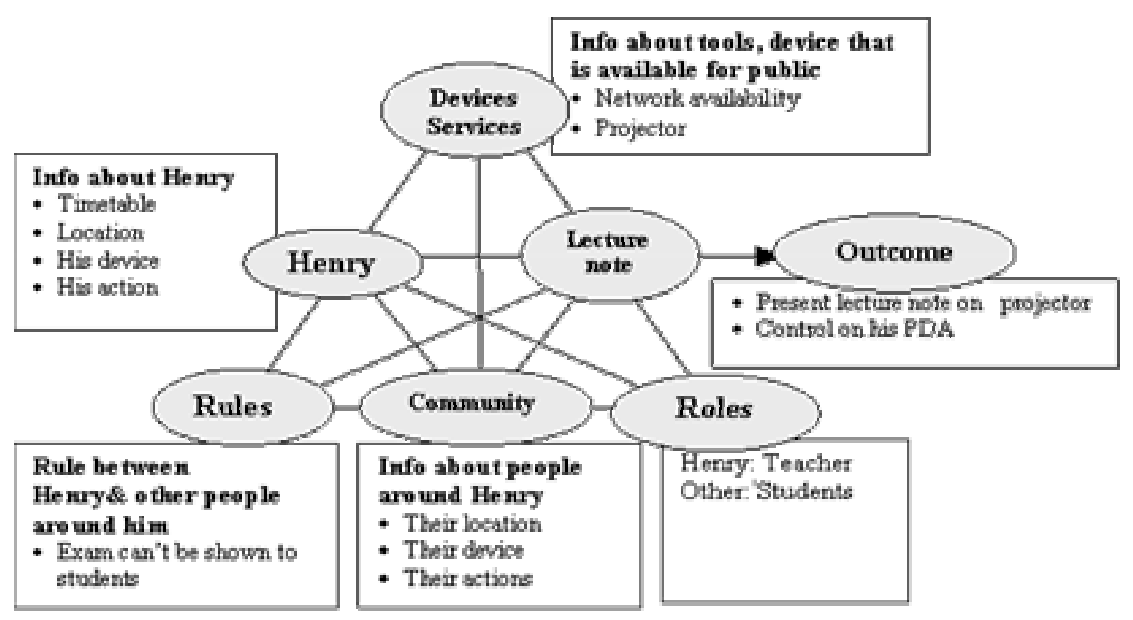

Figure 2. Classify the context in the scenario by using Activity Theory

Figure 2 shows how the context in this scenario is drawn out by the Activity Theory model. The context-aware system presents a selection of files on his PDA based on his current location, time, people around him, his role, rules and tool availability. Once he has selected a file, the system presents the contents on a projector for the students to see and allows Henry to control it via his PDA.

\section{From Activity Theory to Context Model}

The example scenario above briefly illustrates that Activity Theory has the potential to be used as a foundation for producing a (sufficiently) comprehensive context classification system. The elements in Activity Theory cover the key elements of context in the scenario that have an influence on human activity. Moreover, the relationship between each element is also identified. However, this model lacks a representation of history, which can be represented through time.

Time is a crucially important part of context. This includes not just current time, but also past time (that contributes a history element to the context) and future time (that allows for prediction of users' actions from the current context). Hence, we must account for time in our context models. We propose the context model illustrated in Figure 3. The elements in the model can be described as follows:

User: Information about the user and her physical environment that has influence on her activity, including user's current location, action, device and timetable.

Tools and their availability: Tools those are available in the public space and their availability, including device characteristics, public services and computing environment such as network availability. 
Rules: Norms, social rules and legislation within which the user relates to others in her community.

Community: Information about people around the user (in both physical and virtual environments) that may have an influence on her activity.

Division of Labour: Roles of user in that situation including who can perform which tasks on the object.

Object: User's intention and objective. The system uses all the elements above to decide about the user's intention or objective.

Time: For our current purposes, time is the occurrence of events in the past, the present and the future.

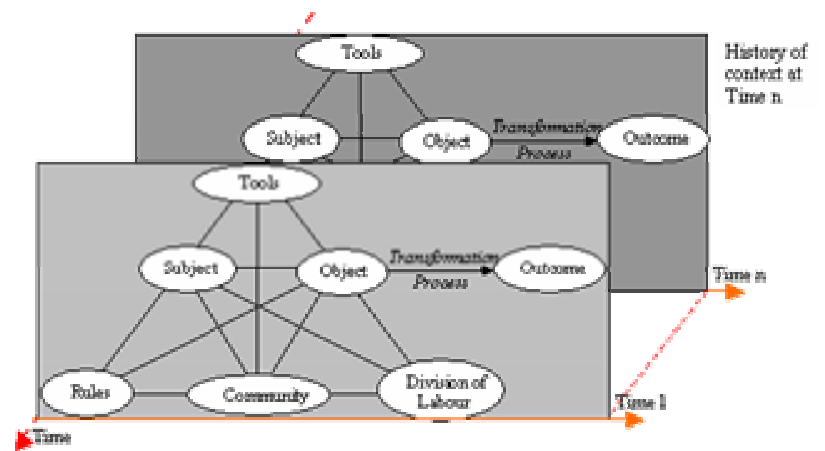

Figure 3. Context model adapted from Activity Theory

This is a first attempt at modelling a comprehensive context classification based on Activity Theory. A cycle of application, evaluation and iteration is required to ensure that the classification covers key elements in context awareness and identifies relevant relationships. Our next step is to generate more scenarios to produce a more comprehensive context classification model and then to evaluate this classification model. This model will then be used as part of the framework for implementing a context aware system. The system will then be tested with real users and evaluated to see if it reduces the user's explicit input and provides the user with a usable contextaware system.

\section{Conclusion}

In this research, we aim to provide a comprehensive context classification system that includes the key elements of context that have an influence on a user's diverse activities in an ambient computing world. We also hope to identify the relationships between each element in the classification so that these relationships may be applied during the development of a context aware system. This model can then be used a framework in the design process as the model will provide a better understanding of context. 


\section{References}

1. Abowd, G. D., et al. (2000). CybreMinder: A Context-Aware System for Supporting Reminders. Proc. of Second Int. Symposium on Handheld and Ubiquitous Computing, HUC 2000, Bristol, UK, Springer Verlag.

2. Benerecetti, M., et al. (2001). "Distributed Context-Aware Systems." HumanComputer Interaction 16(Special issue on Context-aware computing): 213-228.

3. Carroll, J. M. editor. (2000). Making Use: Scenario-Based Design of HumanComputer Interactions. Cambridge, MA, MIT Press.

4. Chalmers, D., et al. (1999). QoS and Context Awareness for Mobile Computing. Handheld and Ubiquitous Computing, First International Symposium, HUC'99, Karlsruhe, Germany, Springer.

5. Chen, G., et al. (2000). A Survey of Context-Aware Mobile Computing Research, Dartmouth College, Department of Computer Science.

6. Dey, A. K., et al. (1999). Toward a Better Understanding of Context and ContextAwareness. Atlanta, GA, USA, Georgia Institute of Technology.

7. Diaper, D., et al. editor. (2003). Handbook of Task Analysis for Human-Computer Interaction, Lawrence Erlbaum Assoc Inc.

8. Engeström, Y., et al., Eds. (1999). Perspectives on Activity Theory. Activity Theory and Individual and Social Transformation, Cambridge University Press.

9. Hull, R., et al. (1997). Towards Situated Computing. Proc. of The First Int. Symposium on Wearable Computers, Cambridge, Massachusetts, IEEE Computer Society Press.

10. Johnson, H., et al. (1991). "Task Knowledge Structures: psychological basis and integration into system design." Acta Psychologica 78: 3-26.

11.Kaptelinin, V., et al. (1997). Activity Theory: Basic Concepts and Application. CHI 1997, Los Angeles.

12.Lieberman, H., et al. (2000). "Out of context: Computer Systems that Adapt to, and Learn from, Context." IBM Systems Journal 39(Nos. 3\&4): 617-632.

13.Lucas, P. (2001). "Mobile Devices and Mobile Data-Issues of Identity and Reference." Human-Computer Interaction 16(2): 323-336.

14. Schmidt, A., et al. (1999). "There is More to Context Than Location." Computers and Graphics 23(6): 893-901. 